\title{
Multivariate Copula Approach for Polarimetric Classification in Weather Radar Applications
}

\author{
F.J. Yanovsky, A.N. Rudiakova, and R.B. Sinitsyn \\ Electronics Department \\ National Aviation University \\ Prospect Komarova 1, 03058, Kiev, UKRAINE \\ yanovsky@nau.edu.ua, anna.rudiakova@gmail.com,rsinitsyn@gmail.com
}

\begin{abstract}
The paper presents a multivariate copula approach to identify the dependence between different polarimetric parameters. This approach can be used to develop a new method of invariant polarimetric classification of radar targets. Signals from meteorological target are processed as an example.
\end{abstract}

Index Terms - Radar signal processing; meteorological radar

\section{INTRODUCTION}

It is known that the most powerful signal processing in weather radar is possible using fully polarimetric measurements. This gives one the couple of orthogonal polarizations' signal samples, which are obtained simultaneously at the same time moments. Therefore, the time series of all combinations of sounding and receiving polarizations would be used to calculate the polarimetric parameters [1-4]. The polarimetric classification of radar data deals with interpretation of mutual dependence of these parameters. The reflectivity, differential reflectivity, crosscorrelation coefficient, specific differential phase, etc. are used for discrimination between the several classes of weather radar echo $[1,5]$. However, the most of classification algorithms are based on the principles of fuzzy logic [6-8].

Recently, the copula cross-correlation and cross-spectra functions were presented $[9,10]$ for the signal processing of polarimetric weather radar. The analysis was given for the bivariate distributions of measured signal pairs. In this paper we introduce a multivariate copula approach that can be used to identify the dependence between the measured scattering matrix elements for the different weather conditions, and therefore would be the possible method for invariant polarimetric classification. Three-dimensional random model of the output signal is used, and vine copulas decompose the multivariate densities into bivariate linking copulas.

\section{MARGINAL DisTRIBUTIONS OF FULL POLARIMETRIC RADAR'S SIGNALS}

In case of the full polarimetric radar, the elements of scattering matrix could be calculated using the measured backscattered signal quadrature components. For the reciprocal medium, the vertical-horizontal and vise-versa polarizations are the same, therefore the only three combinations of the transmit and receive horizontal $(\mathrm{H})$ and vertical $(\mathrm{V})$ polarizations are unique. The polarimetric signals under consideration can be described in the following manner:

$$
\left[\begin{array}{c}
\mathbf{E}_{H}^{s} \\
\mathbf{E}_{V}^{s}
\end{array}\right]=[\mathbf{S}]\left[\begin{array}{l}
\mathbf{E}_{H}^{i} \\
\mathbf{E}_{V}^{i}
\end{array}\right]=\left[\begin{array}{ll}
\mathbf{S}_{H H} & \mathbf{S}_{H V} \\
\mathbf{S}_{V H} & \mathbf{S}_{V V}
\end{array}\right]\left[\begin{array}{l}
\mathbf{E}_{H}^{i} \\
\mathbf{E}_{V}^{i}
\end{array}\right],
$$

where the $i$ and $s$ superscripts means the incident and scattered fields, respectively.

In this paper, we propose the analysis of complex data measured at the output of the full polarimetric radar (PARSAX [11]). After some initial processing, the co- and cross-polar components of scattering matrix can be obtained as the time series of complex signals $\left\{\mathbf{S}_{H H}, \mathbf{S}_{H V}, \mathbf{S}_{V V}\right\}$ for the each of range bins. The absolute values of these signals $\left\{S_{H H}, S_{H V}, S_{V V}\right\}$, respectively, are used hereinafter. This triplet can be transformed to the uniformly distributed one:

$$
\begin{gathered}
S_{H H}^{U F}=F_{H H}\left(S_{H H}\right), \quad S_{H V}^{U F}=F_{H V}\left(S_{H V}\right), \\
S_{V V}^{U F}=F_{V V}\left(S_{V V}\right),
\end{gathered}
$$

where $F_{H H}\left(S_{H H}\right), F_{H V}\left(S_{H V}\right)$ and $F_{V V}\left(S_{V V}\right)$ are the corresponding cumulative distribution functions, used as marginal distributions.

\section{Multivariate COPUla AND COPUla DENSITY}

The multivariate cumulative distribution function (CDF) of the transformed variables $\left\{S_{H H}^{U F}, S_{H V}^{U F}, S_{V V}^{U F}\right\}$ has name of a copula and as a result of the Sklar's theorem can be written in the form

$$
\begin{gathered}
F\left(S_{H H}, S_{H V}, S_{V V}\right)= \\
=C\left(F_{H H}\left(S_{H H}\right), F_{H V}\left(S_{H V}\right), F_{V V}\left(S_{V V}\right)\right),
\end{gathered}
$$

where $F\left(S_{H H}, S_{H V}, S_{V V}\right)$ is a multivariate cumulative distribution function of $\left\{S_{H H}, S_{H V}, S_{V V}\right\}$. The multivariate copula, furthermore, can be represented as the linked set of 
bivariate copulas, so called, vine copula [12-14]. For the 3-D vine copula one can construct the canonical C-vine, or D-vine, that can be represented using trees $T_{1}-T_{2}$, as shown in Fig. 1.
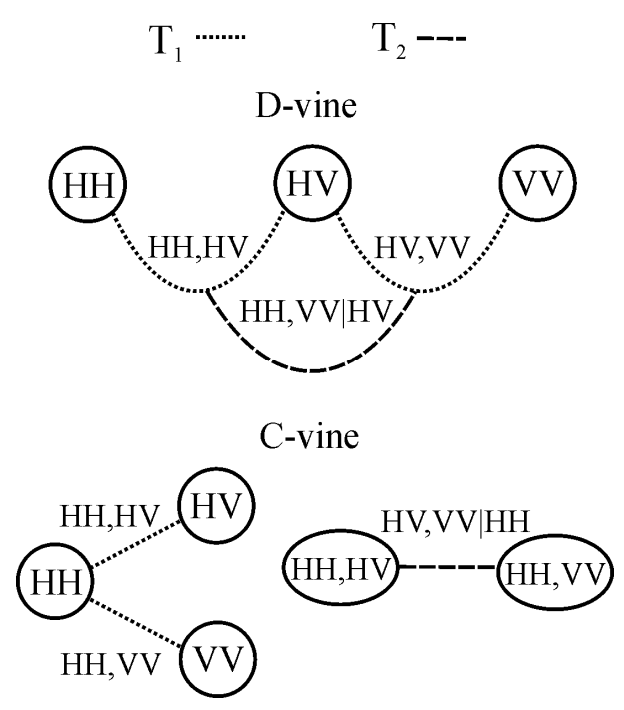

Figure 1. C-vine and D-vine representations for the 3-D vine copula

Multivariate probability density function (PDF) can be expressed as the $3^{\text {rd }}$ derivative of multivariate CDF as follows:

$$
\begin{gathered}
f\left(S_{H H}, S_{H V}, S_{V V}\right)= \\
=\frac{\partial^{3} C\left(F_{H H}\left(S_{H H}\right), F_{H V}\left(S_{H V}\right), F_{V V}\left(S_{V V}\right)\right)}{\partial S_{H H} \partial S_{H V} \partial S_{V V}}= \\
=\frac{\partial^{3} C\left(S_{H H}^{U F}, S_{H V}^{U F}, S_{V V}^{U F}\right)}{\partial S_{H H}^{U F} \partial S_{H V}^{U F} \partial S_{V V}^{U F}} \times \\
\times \frac{d F_{H H}\left(S_{H H}\right)}{d S_{H H}} \cdot \frac{d F_{H V}\left(S_{H V}\right)}{d S_{H V}} \cdot \frac{d F_{V V}\left(S_{V V}\right)}{d S_{V V}}= \\
=c\left(F_{H H}\left(S_{H H}\right), F_{H V}\left(S_{H V}\right), F_{V V}\left(S_{V V}\right)\right) \times \\
\times f_{H H}\left(S_{H H}\right) \cdot f_{H V}\left(S_{H V}\right) \cdot f_{V V}\left(S_{V V}\right),
\end{gathered}
$$

where $c\left(F_{H H}\left(S_{H H}\right), F_{H V}\left(S_{H V}\right), F_{V V}\left(S_{V V}\right)\right)$ is a multivariate copula density function, $f_{H H}\left(S_{H H}\right), f_{H V}\left(S_{H V}\right), f_{V V}\left(S_{V V}\right)$ are the marginal densities. According to the D-vine trees in Fig. 1, the multivariate copula density can be represented by the bivariate functions in following manner:

$$
\begin{gathered}
f\left(S_{H H}, S_{H V}, S_{V V}\right)= \\
=c_{H H, H V}\left(S_{H H}^{U F}, S_{H V}^{U F}\right) \cdot c_{H V, V V}\left(S_{H V}^{U F}, S_{V V}^{U F}\right) \times \\
\times c_{H H, V V \mid H V}\left(F_{H H \mid H V}\left(S_{H H} \mid S_{H V}\right), F_{V V \mid H V}\left(S_{V V} \mid S_{H V}\right)\right) \times \\
f_{H H}\left(S_{H H}\right) \cdot f_{H V}\left(S_{H V}\right) \cdot f_{V V}\left(S_{V V}\right),
\end{gathered}
$$

where the $c_{i, j}(i, j \rightarrow H H, H V$, or $V V)$ is corresponding bivariate copula density function, $c_{H H, V V \mid H V}(\cdot)$ is the conditional bivariate copula density function, and $F_{i \mid j}$ is expressed as follows:

$$
F_{i \mid j}\left(S_{i} \mid S_{j}\right)=\frac{\partial C_{i, j}\left(F_{i}\left(S_{i}\right), F_{j}\left(S_{j}\right)\right)}{\partial F_{j}\left(S_{j}\right)} .
$$

As can be seen from (2), the multivariate PDF can be represented by the set of bivariate copula functions, that contains the pure dependence, and a set of the marginal distributions.

For the measured polarimetric signals the kernel estimates should be used instead of their original CDF, PDF, copulas and copula densities. It is supposed, that the estimates are converging to the original functions with increasing the number of samples.

\section{DATA PROCESSING AND RESUlTS}

Application of proposed multivariate copula based dependence measure approach can be shown using the data received by the PARSAX radar. The sounding signal of this radar can have vertical or horizontal polarizations and also there is a possibility to send this signal in the same direction during many periods of observation. The considering data sets for $\mathrm{HH}, \mathrm{HV}$ and VV polarizations, particularly, contain 512 periods. The areas of radar observation that contain the precipitation regions are shown in Fig. 2, 3 for the wet snow (Fig. 2) and the rain thunderstorm (Fig. 3).

The original and transformed polarization signals' time series according to the both cases of weather conditions are shown in Fig. 4 (wet snow) and Fig. 5 (rain thunderstorm).

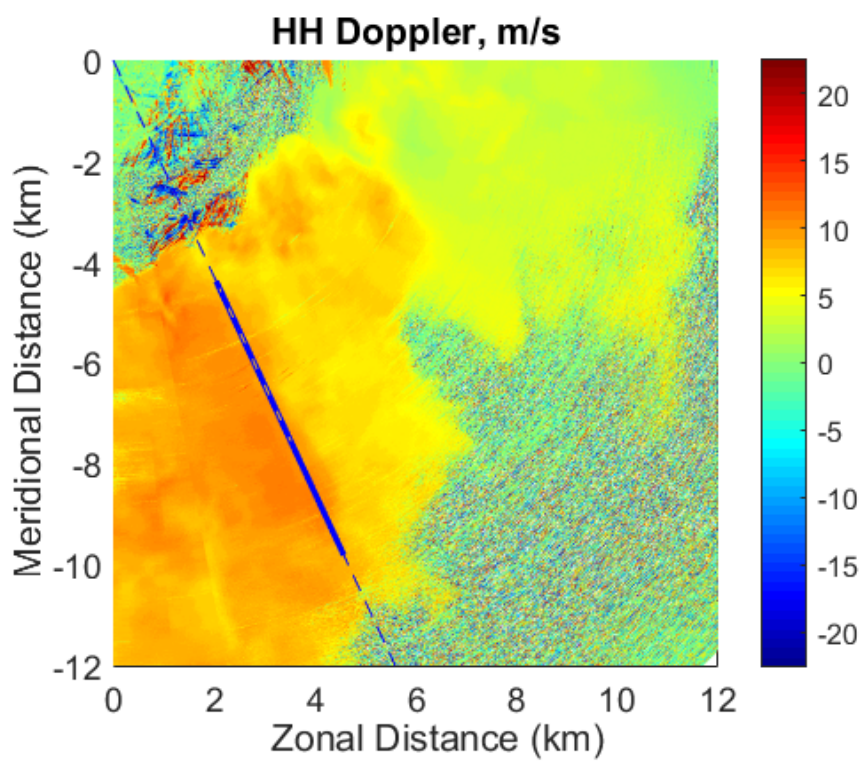

Figure 2. The considering region with wet snow. Dashed line shows the processing direction (azimuth $155^{\circ}$ ), while bold solid line shows the processing range bins. 


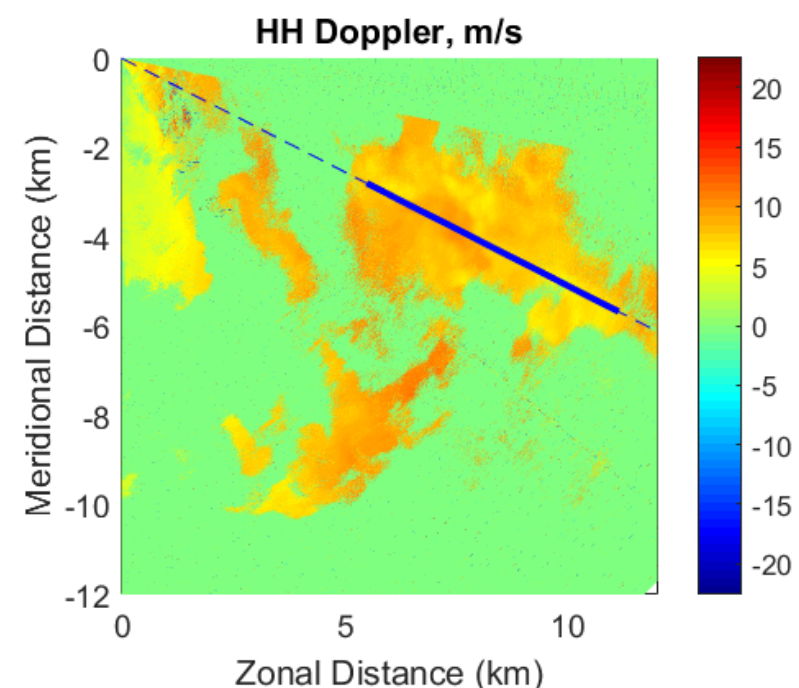

Figure 3. The considering region with rain thunderstorm. Dashed line shows the processing direction (azimuth $117^{\circ}$ ), while bold solid line shows the processing range bins.
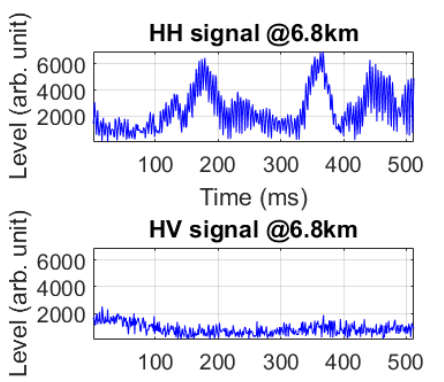

Time (ms)
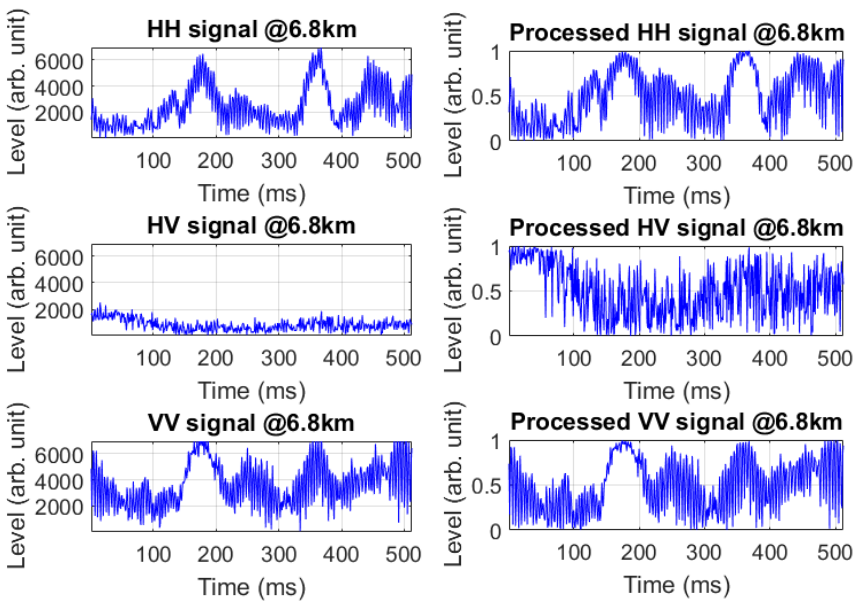

Time (ms)

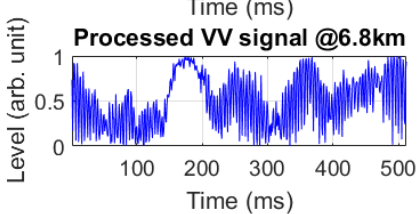

Figure 4. Measured polarization signals' time series for wet snow weather conditions.
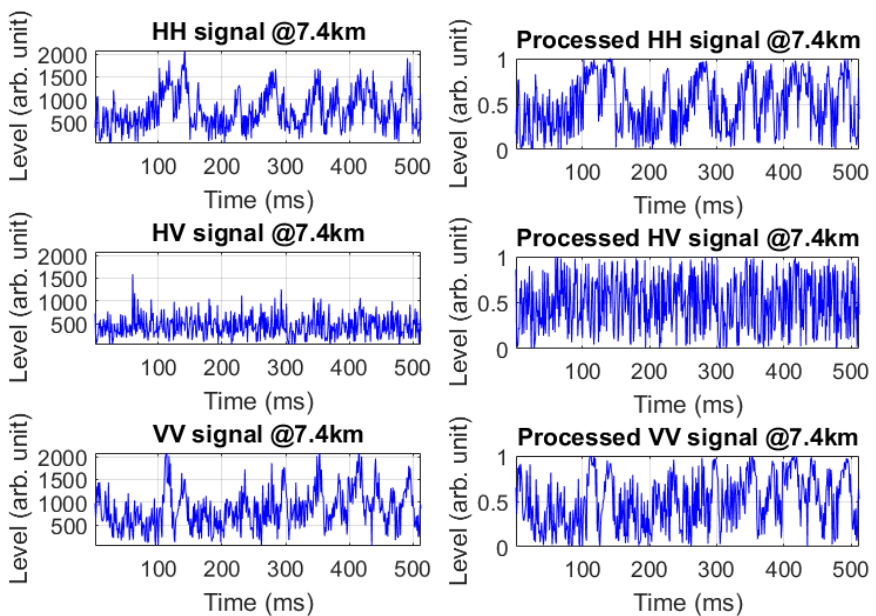

Figure 5. Measured polarization signals' time series for rain thunderstorm weather conditions
The 2-D distributions of original polarization signals and copula distributions are shown for the selected pairs of $\mathrm{HH}$, HV and VV components in Fig. 6 (wet snow) and Fig. 7 (rain thunderstorm).

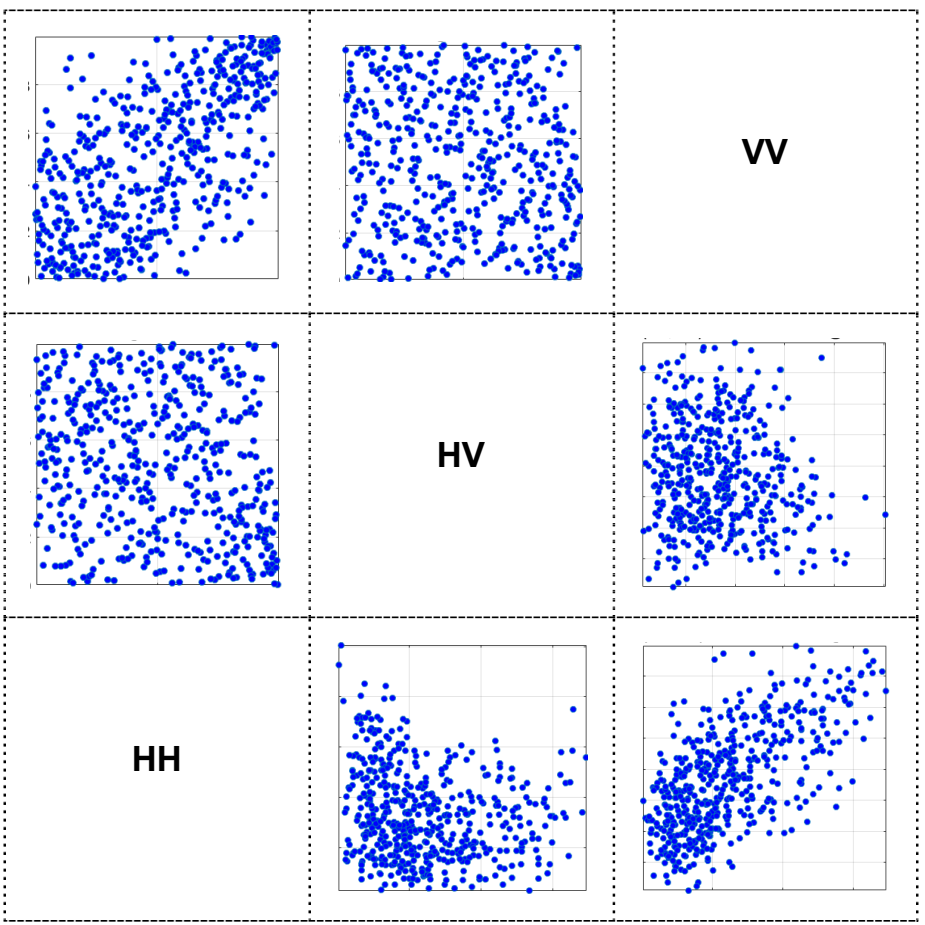

Figure 6. The copula distributions (left upper pictures) and 2-D distributions of original polarization signals (right bottom pictures) for the (HH,VV), (HV, $\mathrm{HH})$ and $(\mathrm{HV}, \mathrm{VV})$ pairs and the wet snow weather conditions.
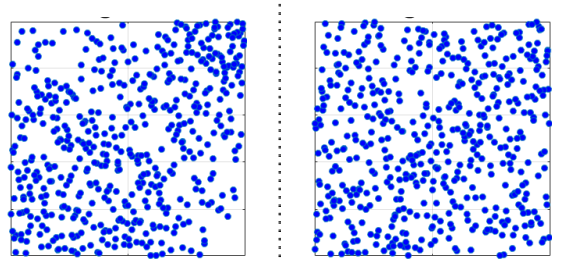

V

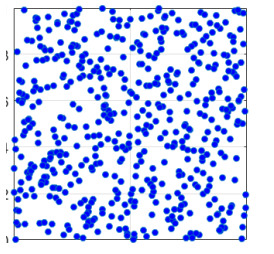

HV

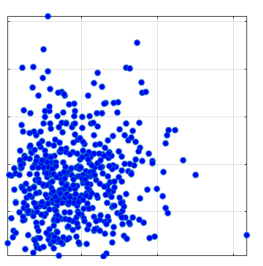

HH
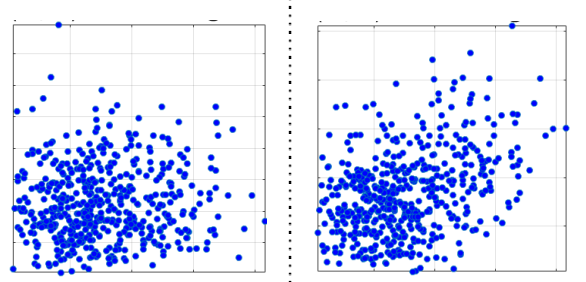

Figure 7. The copula distributions (left upper pictures) and 2-D distributions of original polarization signals (right bottom pictures) for the (HH,VV), (HV, $\mathrm{HH})$ and $(\mathrm{HV}, \mathrm{VV})$ pairs and the rain thunderstorm weather conditions. 
The copula and copula densities functions are shown for the selected pairs of $\mathrm{HH}, \mathrm{HV}$ and VV components in Fig. 8 (wet snow) and Fig. 9 (rain thunderstorm).

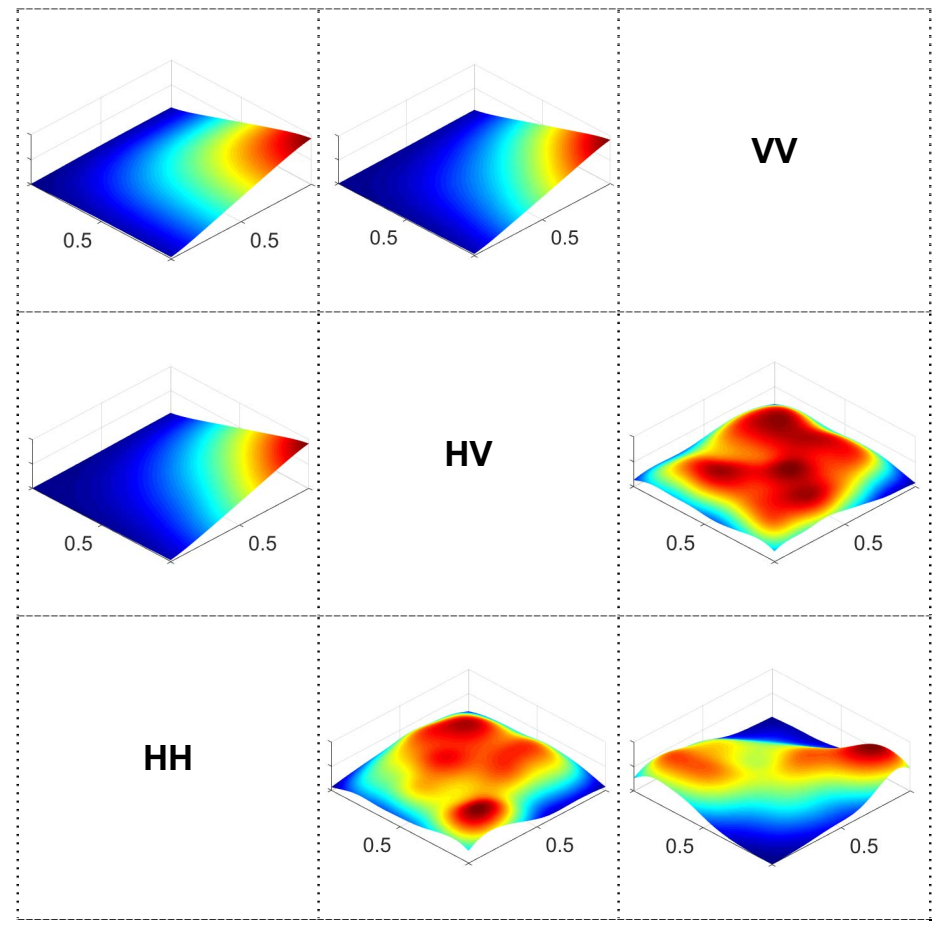

Figure 8. The copula (left upper pictures) and copula density (right bottom pictures) functions for the $(\mathrm{HH}, \mathrm{VV}),(\mathrm{HV}, \mathrm{HH})$ and $(\mathrm{HV}, \mathrm{VV})$ pairs and the wet snow weather conditions.

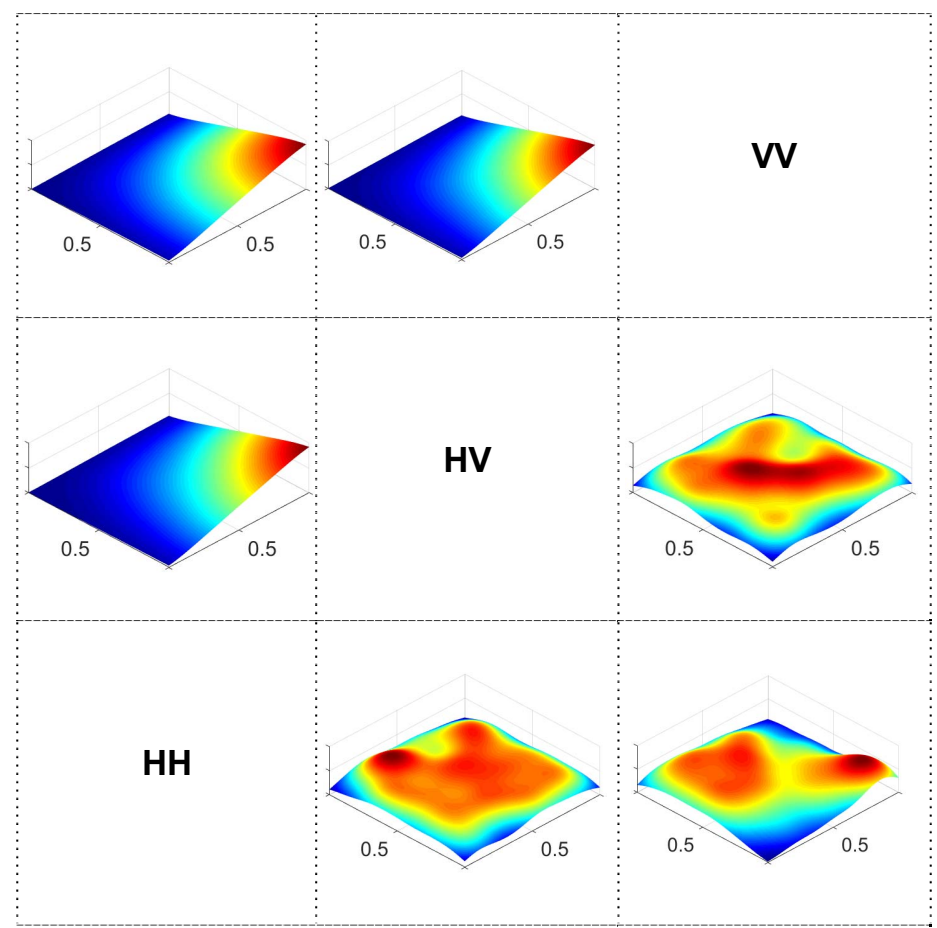

Figure 9. The copula (left upper pictures) and copula density (right bottom pictures) functions for the $(\mathrm{HH}, \mathrm{VV}),(\mathrm{HV}, \mathrm{HH})$ and $(\mathrm{HV}, \mathrm{VV})$ pairs and the rain thunderstorm weather conditions.
As one can see from a comparison of Figures 6 and 7, the pairwise two-dimensional distributions of polarization components $\mathrm{HH}, \mathrm{HV}$ and $\mathrm{VV}$ are quite significantly different in cases of wet snow and heavy rain. This result can be used in the classification of hydrometeors. It is worthy to amfisize that the pointed differences appear most clearly for the twodimensional density copula distributions (Figs. 8 and 9). This result is an expected one because, as it follows from (1), the structures of relationships between considered distributuions are contained exactly in two-dimensional copula distributions.

For the further development of research in the field of classification based on the analysis of multivariate copulas, we need to introduce quantitative methods for evaluating the links, for example, based on the Bayesian approach that will allow developing the algorithms suitable for the automation of the classification process.

We also plan to combine copula signal processing with modeling especially for the case of precipitation surveillance taking into account the inertia of scatterers [15].

It is reasonable to note that the proposed multivariate copula approach for polarimetric classification is suitable not only for meteorological application, which has been considered as an example, but also for many other applications including ultra-wideband signals processing [16], in particular for through-the-wall surveillance technologies [17].

\section{CONCLUSION}

The set of the introduced new linked bivariate copulas, in the theory, fully represents the dependence structure of their marginal distributions. This behavior can be utilized for the invariant polarimetric classification of the objects. Further investigations are necessary, both theoretical and experimental, in order to design really useful and practically applicable invariant classification methods and algorithms.

\section{ACKNOWLEDGMENT}

The authors are very grateful to the Delft University of Technology (TU-Delft), the Netherlands, and personally to Prof. Alex Yarovoy and Dr. Oleg Krasnov for providing the measurement data of meteorological observations with PARSAX, the programmable Polarimetric Agile Radar in Sand X-band.

\section{REFERENCES}

[1] V.N. Bringi and V. Chandrasekar, Polarimetric Doppler Weather Radar. Cambridge, U.K.: Cambridge Univ. Press, 2002.

[2] R. J. Doviak and D. S. Zrnic, Doppler Radar and Weather Observations, 2nd ed. San Diego, CA: Academic, 1993.

[3] F. Yanovsky, "Inferring microstructure and turbulence properties in rain through observations and simulations of signal spectra measured with Doppler-polarimetric radars", Book Chapter in: Polarimetric Detection, Characterization, and Remote Sensing, Springer, 2011, p. 501-545. 
[4] Ryzhkov, A. V., 2001: Interpretation of polarimetric radar covariance matrix for meteorological scatterers: theoretical analysis. J. Atmos. Oceanic Technol. 18, 315-328.

[5] F.J. Yanovsky, H.W.J. Russchenberg, L.P. Ligthart, V.S. Fomichev, Microwave Doppler-polarimetric technique for study of turbulence in precipitation, IEEE International Geoscience and Remote Sensing Symposium (IGARSS), 2000, pp. Vol. V, pp. 2296-2298.

[6] H. Liu, V. Chandrasekar, "Classification of Hydrometeors Based on Polarimetric Radar Measurements: Development of Fuzzy Logic and Neuro-Fuzzy Systems, and In Situ Verification”, J. Atmos. Oceanic Technol., 17, 140-164.

[7] Hyang Suk Park, A. V. Ryzhkov, D. S. Zrnić, and Kyung-Eak Kim, 2009: The Hydrometeor Classification Algorithm for the Polarimetric WSR-88D: Description and Application to an MCS. Wea. Forecasting, 24, 730-748.

[8] Ya. P. Ostrovsky, F. J. ,Yanovsky, Precipitation and turbulence intensity classification based on the polarimetric doppler radar data analysis, Telecommunications and Radio Engineering, Vol. 65, No 12, pp. 1077 1085. DOI: 10.1615/TelecomRadEng.v65.i12.20.

[9] A.A. Pitertsev, R.B. Sinitsyn, F.J. Yanovsky, Copula Based Dependence Measure for Polarimetric Weather Radar, Proc. International Radar Symposium (IRS-2015), Dresden, Germany, 2015, pp. 597-603.

[10] A.N. Rudiakova, R. B. Sinitsyn and F. J. Yanovsky, Invariant polarimetric parameters for signal processing in weather radar, The International IEEE Conference on Microwaves, Communications Antennas and Electronic Systems, Tel-Aviv, Israel, November 2-4, 2015, $6 \mathrm{pp}$.

[11] O.A. Krasnov, L.P. Ligthart, G.P. Babur, and F. van der Zwan, The PARSAX - New Full Polarimetric FMCW Radar with Dual-Orthogonal
Signals, Proceedings of the 8th International Symposium on Tropospheric Profiling, ISBN 978-90-6960-233-2, Delft, The Netherlands, October 2009. Editors, A. Apituley, H.W.J. Russchenberg, W.A.A. Monna. B 25, 2009, pp. S06-P08-1-S06-P08-4.

[12] Y. Gyasi-Agyei, Copula - based daily rainfall disaggregation model, Water Resour. Res., 47, W07535, 2011.

[13] H. Joe, Families of m-variate distributions with given margins and $\mathrm{m}(\mathrm{m}$ - 1)/2 bivariate dependence parameters, in Distributions With Fixed Marginals and Related Topics, Lect. Notes Monogr. Ser., vol. 28, edited by L. Ruschendorf, B. Schweizer, and M. D. Taylor, 1996, pp. 120-141, Inst. of Math. Stat., Hayward, Calif.

[14] K. Aas, C. Czado, A. Frigessi, and H. Bakken, Pair - copula constructions of multiple dependence, Insur. Math. Econ., 44, 2009, pp. 182-198.

[15] F. J. Yanovsky, Simulation study of $10 \mathrm{GHz}$ radar backscattering from clouds, and solution of the inverse problem of atmospheric turbulence measurements, Computation in Electromagnetics, Conf. publication, IEE (UK) No. 420, 1996, pp. 188-193. DOI: 10.1049/cp:19960182.

[16] F. J. Yanovsky, R. B. Sinitsyn, Ultrawideband signal processing algorithms for radars and sodars, Proceedings Third International Conference on Ultrawideband and Ultrashort Impulse Signals (UWBUSIS 2006), September 18-22, 2006, Sevastopol, Ukraine, pp. 6671. DOI: 10.1109/UWBUS.2006.307160.

[17] F. J. Yanovsky, V. E. Ivashchuk, V. P. Prokhorenko, Through-the-wall surveillance technologies, Proceedings 2012 6th International Conference on Ultrawideband and Ultrashort Impulse Signals (UWBUSIS 2012), 17-21 September, 2012, Sevastopol, Ukraine, pp. 3033. DOI: 10.1109/UWBUSIS.2012.6379723. 